\title{
Exact Solutions of a High-Order Nonlinear Wave Equation of Korteweg-de Vries Type under Newly Solvable Conditions
}

\author{
Weiguo Rui \\ College of Mathematics, Chongqing Normal University, Chongqing 401331, China \\ Correspondence should be addressed to Weiguo Rui; weiguorhhu@aliyun.com
}

Received 31 October 2013; Accepted 7 January 2014; Published 4 March 2014

Academic Editor: Zuo-nong Zhu

Copyright (C) 2014 Weiguo Rui. This is an open access article distributed under the Creative Commons Attribution License, which permits unrestricted use, distribution, and reproduction in any medium, provided the original work is properly cited.

\begin{abstract}
By using the integral bifurcation method together with factoring technique, we study a water wave model, a high-order nonlinear wave equation of KdV type under some newly solvable conditions. Based on our previous research works, some exact traveling wave solutions such as broken-soliton solutions, periodic wave solutions of blow-up type, smooth solitary wave solutions, and nonsmooth peakon solutions within more extensive parameter ranges are obtained. In particular, a series of smooth solitary wave solutions and nonsmooth peakon solutions are obtained. In order to show the properties of these exact solutions visually, we plot the graphs of some representative traveling wave solutions.
\end{abstract}

\section{Introduction}

In this work, we will study the following high-order nonlinear wave equation of Korteweg-de Vries type:

$$
\begin{aligned}
\eta_{t} & +\eta_{x}+\alpha \eta \eta_{x}+\beta \eta_{x x x}+\rho_{1} \alpha^{2} \eta^{2} \eta_{x} \\
& +\alpha \beta\left(\rho_{2} \eta \eta_{x x x}+\rho_{3} \eta_{x} \eta_{x x}\right)+\rho_{4} \alpha^{3} \eta^{3} \eta_{x} \\
& +\alpha^{2} \beta\left(\rho_{5} \eta^{2} \eta_{x x x}+\rho_{6} \eta \eta_{x} \eta_{x x}+\rho_{7} \eta_{x}^{3}\right)=0 .
\end{aligned}
$$

This is an important model of water wave derived by Fokas [1] in 1995, where $\alpha=3 A / 2, \beta=B / 6$, and $0<\alpha, \beta \ll 1$.

Obviously, (1) is a very complex partial deferential equation, it has nine parameters $\alpha, \beta, \rho_{i},(i=1,2, \ldots, 7)$, and contains both high-order derivative terms and multinonlinear terms. It is very different from the original KdV equation. Regarding the $\rho_{1}, \rho_{2}, \rho_{3}, \rho_{4}, \rho_{5}, \rho_{6}, \rho_{7}$ as free parameters, Tzirtzilakis et al. [2] investigated solitary wave solutions of (1) and they called (1) high-order wave equation of Korteweg-de Vries type. Just as Tzirtzilakis et al. [2] said that investigations of solitary wave solutions of (1) are more physically and practically meaningful. The motion described by the model (1) is a 2-dimensional, inviscid, and incompressible fluid (water) lying above a horizontal flat bottom located at $\bar{y}=$ $-\hbar_{0}\left(\hbar_{0}\right.$ is a constant) and letting the air above the water. It turns out that, for such a system if the vorticity is zero initially, it remains zero. The fluids (waters) analyzed by Fokas are only irrotational flows. This system is characterized by two parameters $\alpha=3 A / 2$ and $\beta=B / 6$ with $A=a / \hbar_{0}$ and $B=\hbar_{0}^{2} / \ell^{2}$, where $a$ and $\ell$ are two typical values of the amplitude and of the wavelength of the waves. The parameters $a, \hbar_{0}$, and $\ell$ satisfy the condition $a \ll \hbar_{0}<\ell$ because the system is a model of short amplitude and long wavelength.

When $\rho_{1}=\rho_{2}=\rho_{3}=\rho_{4}=\rho_{5}=\rho_{6}=\rho_{7}=0$, (1) can be reduced to the classical (original) KdV equation:

$$
\eta_{t}+\eta_{x}+\alpha \eta \eta_{x}+\beta \eta_{x x x}=0 .
$$

In [1], Fokas assumed that $O(\beta)<O(\alpha)$ and $\beta \approx \alpha^{2}$. According to this assumption, we easily know that $O\left(\alpha^{2} \beta\right)<O\left(\alpha^{3}\right)$ and $O\left(\alpha^{2} \beta\right)<O(\alpha \beta)$. Neglecting two high-order infinitesimal terms of $O\left(\alpha^{3}, \alpha^{2} \beta\right)$, (1) can be reduced to another high-order wave equation of $\mathrm{KdV}$ type [2-5] as follows:

$$
\begin{gathered}
\eta_{t}+\eta_{x}+\alpha \eta \eta_{x}+\beta \eta_{x x x}+\rho_{1} \alpha^{2} \eta^{2} \eta_{x} \\
+\alpha \beta\left(\rho_{2} \eta \eta_{x x x}+\rho_{3} \eta_{x} \eta_{x x}\right)=0 .
\end{gathered}
$$

Equation (3) can be regarded as a special case of (1) for $\rho_{4}=$ $\rho_{5}=\rho_{6}=\rho_{7}=0$. In [1], it was observed that (3) can be reduced by the local transformation of coordinates

$$
\eta=v-\alpha \rho_{1} v^{2}-\beta\left(3 \rho_{1}+\frac{7}{4} \rho_{2}-\frac{1}{2} \rho_{3}\right) v_{x x}
$$


to a completely integrable PDE as follows:

$$
\begin{aligned}
v_{t} & -\frac{3}{2} \beta \rho_{2} v_{x x t}+\beta\left(1-\frac{3}{2} \rho_{2}\right) v_{x x x}+\alpha v v_{x} \\
& -\frac{1}{2} \alpha \beta \rho_{2}\left(v v_{x x x}+2 v_{x} v_{x x}\right) .
\end{aligned}
$$

Equation (5) was first derived in [6] by using the method of bi-Hamiltonian systems and its Lax pair was given in [7].

Neglecting the highest-order infinitesimal term of $O\left(\alpha^{2} \beta\right)$, (1) can be reduced to a new generalized $\mathrm{KdV}$ equation as follows:

$$
\begin{aligned}
\eta_{t} & +\eta_{x}+\alpha \eta \eta_{x}+\beta \eta_{x x x}+\rho_{1} \alpha^{2} \eta^{2} \eta_{x} \\
& +\alpha \beta\left(\rho_{2} \eta \eta_{x x x}+\rho_{3} \eta_{x} \eta_{x x}\right)+\rho_{4} \alpha^{3} \eta^{3} \eta_{x}=0
\end{aligned}
$$

We call it a generalized $\mathrm{KdV}$ equation of neglecting the highest-order infinitesimal term [8]. In fact, (6) can be regarded as another special case of (1) for $\rho_{5}=\rho_{6}=\rho_{7}=0$; it is also third-order approximate equation of higher-order $\mathrm{KdV}$ type.

From the above references and the references cited therein, we know that (1) is a very important model of water wave. However, (1) is too complex to obtain its exact solution under universal conditions. Only under some very special parametric conditions, its exact solutions were obtained in existing literatures [2-5, 8-12]. In addition, in [13], under different kinds of parametric conditions, Marinakis discussed two integrable cases for the third-order approximation model (1). In [14], Marinakis proved that (1) and its some special cases are integrable. Generally, a system is regarded as that it is integrable if it has Darboux transformation, Lax pair, bilinear structure and multilinear structure, Hamilton function, first integral function (equation), symmetrical structure (i.e., symmetry), conservation law, and so forth. In [15], Gandarias and Bruzon proved that (1) is self-adjoint if and only if $\rho_{3}=$ $2 \rho_{2}$, and $\rho_{7}=\rho_{6}-3 \rho_{5}$.

From the above research backgrounds of (1), we can see that its exact solutions under universal conditions are hard to obtain because it is highly nonlinear equation and most probably it is not integrable equation in general. Thus, large numbers of research results are still concentrated on the generalized $\mathrm{KdV}, \mathrm{mKdV}$ equations $[16,17]$ and other some high-order equations with $\mathrm{KdV}$ type, such as KdV-Burgers equation [18, 19] and KdV-Burgers-Kuramoto equation [20]. Therefore, studying solvability and finding exact solutions of (1) within more extensive parameter ranges are very important and necessary. In this paper, based on the works in [8], by using the integral bifurcation method [21], we will investigate solvable conditions and exact traveling wave solutions of (1) within more extensive parameter ranges. It is different from those special cases which were considered by authors in existing literatures; we will discuss a newly solvable case $\rho_{3}=(m+1) \rho_{2}, \rho_{6}=2(m+1) \rho_{5}$, and $\rho_{7}=$ $m \rho_{5}, m \in \mathbf{N}$ for (1); indeed, (1) is solvable under these parametric conditions. Though this is still a special case for (1), it is more general than the cases on the assumptions of parametric conditions which appeared in existing literatures.
In particular, the results which will be obtained in this paper are very new and different from those in $[8,9]$.

The rest of this paper is organized as follows. In Section 2, we will derive two-dimensional dynamical system which is equivalent to (1) and give its first integrals. In Section 3, by using the integral bifurcation method, we will investigate different kinds of exact traveling wave solutions of (1) within more extensive parameter ranges and discuss their dynamic properties.

\section{The First Integrals of (1) under Newly Integrable Conditions}

Making a transformation $\eta(x, t)=\phi(\xi)$ with $\xi=x-c t$, (1) can be reduced to the following ODE:

$$
\begin{aligned}
& (1-c) \phi^{\prime}+\alpha \phi \phi^{\prime}+\beta \phi^{\prime \prime \prime}+\rho_{1} \alpha^{2} \phi^{2} \phi^{\prime} \\
& \quad+\alpha \beta\left(\rho_{2} \phi \phi^{\prime \prime \prime}+\rho_{3} \phi^{\prime} \phi^{\prime \prime}\right)+\rho_{4} \alpha^{3} \phi^{3} \phi^{\prime} \\
& \quad+\alpha^{2} \beta\left[\rho_{5} \phi^{2} \phi^{\prime \prime \prime}+\rho_{6} \phi \phi^{\prime} \phi^{\prime \prime}+\rho_{7}\left(\phi^{\prime}\right)^{3}\right]=0
\end{aligned}
$$

where $\phi^{\prime}=d \phi / d \xi, c$ is a wave velocity which moves along the direction of $x$-axis, and $c \neq 0$. It is easy to find that (7) can be integrated once under the conditions $\rho_{3}=(m+1) \rho_{2}$, $\rho_{6}=2(m+1) \rho_{5}$, and $\rho_{7}=m \rho_{5}$, where $m \in \mathbf{N}$. These are newly solvable conditions and they are different from others in $[8,9,13-15]$, and the ranges of parametric values are more extensive than others in existing references. Thus, under these solvable conditions, integrating (7) once and setting the integral constant as zero yield

$$
\begin{aligned}
& (1-c) \phi+\frac{1}{2} \alpha \phi^{2}+\beta \phi^{\prime \prime}+\frac{1}{3} \rho_{1} \alpha^{2} \phi^{3}+\frac{1}{4} \rho_{4} \alpha^{3} \phi^{4} \\
& +\rho_{2} \alpha \beta\left[\phi \phi^{\prime \prime}+\frac{1}{2} m\left(\phi^{\prime}\right)^{2}\right] \\
& +\rho_{5} \alpha^{2} \beta\left[\phi^{2} \phi^{\prime \prime}+m \phi\left(\phi^{\prime}\right)^{2}\right]=0 .
\end{aligned}
$$

When $1+\alpha \rho_{2} \phi+\alpha^{2} \rho_{5} \phi^{2} \neq 0$, (8) can be reduced to a singular two-dimensional system as follows:

$$
\begin{gathered}
\frac{d \phi}{d \xi}=y \\
\frac{d y}{d \xi}=\left(12(c-1) \phi-6 \alpha \phi^{2}-4 \rho_{1} \alpha^{2} \phi^{3}-3 \rho_{4} \alpha^{3} \phi^{4}\right. \\
\left.-6 m \alpha \beta \rho_{2} y^{2}-12 m \alpha^{2} \beta \rho_{5} \phi y^{2}\right) \\
\times\left(12 \beta\left(1+\alpha \rho_{2} \phi+\alpha^{2} \rho_{5} \phi^{2}\right)\right)^{-1}
\end{gathered}
$$

where $\phi^{\prime}=d \phi / d \xi=y$. However, when $1+\alpha \rho_{2} \phi+\alpha^{2} \rho_{5} \phi^{2}=$ $0,(9)$ is not equivalent to (8). In order to obtain a system which is equivalent to (8), we make the following scalar transformation

$$
d \xi=12 \beta\left(1+\alpha \rho_{2} \phi+\alpha^{2} \rho_{5} \phi^{2}\right) d \tau
$$


Equation (9) can be changed into a regular two-dimensional system as follows:

$$
\begin{gathered}
\frac{d \phi}{d \tau}=12 \beta\left(1+\alpha \rho_{2} \phi+\alpha^{2} \rho_{5} \phi^{2}\right) y, \\
\frac{d y}{d \tau}=12(c-1) \phi-6 \alpha \phi^{2}-4 \rho_{1} \alpha^{2} \phi^{3}-3 \rho_{4} \alpha^{3} \phi^{4} \\
-6 m \alpha \beta \rho_{2} y^{2}-12 m \alpha^{2} \beta \rho_{5} \phi y^{2}
\end{gathered}
$$

where $\tau$ is a parameter. Thus, (11) is equivalent to (8). Obviously, (11) and (9) have the same one first integral as follows:

$$
\begin{aligned}
\left(1+\alpha \rho_{2} \phi+\alpha^{2} \rho_{5} \phi^{2}\right) y^{2} & \\
=\frac{1}{6 \beta} \int[ & 12(c-1) \phi+6 \alpha \phi^{2}-4 \rho_{1} \alpha^{2} \phi^{3} \\
& \left.-3 \rho_{4} \alpha^{3} \phi^{4}\right]\left(1+\alpha \rho_{2} \phi+\alpha^{2} \rho_{5} \phi^{2}\right)^{m-1} d \phi .
\end{aligned}
$$

It is easy to find that the integral of right side of (12) is always integrable once the $m$ is given by an idiographic integer. In fact, the first integrals obtained by (12) have certain determinate orderliness; see the following discussions.

(i) When $m=1,(12)$ can be reduced to

$$
\begin{aligned}
y^{2}= & \left(\phi ^ { 2 } \left[(c-1)-\left(\frac{1}{3}\right) \alpha \phi-\left(\frac{1}{6}\right) \rho_{1} \alpha^{2} \phi^{2}\right.\right. \\
& \left.\left.-\left(\frac{1}{10}\right) \rho_{4} \alpha^{3} \phi^{3}\right]+h_{1}\right) \\
& \times\left(\beta\left(1+\alpha \rho_{2} \phi+\alpha^{2} \rho_{5} \phi^{2}\right)\right)^{-1}
\end{aligned}
$$

where $h_{1}$ is an integral constant.

(ii) When $m=2$, (12) can be reduced to

$$
y^{2}=\frac{\phi^{2}\left[(c-1)+a_{1} \phi+a_{2} \phi^{2}+a_{3} \phi^{3}+a_{4} \phi^{4}+a_{5} \phi^{5}\right]+h_{2}}{\beta\left(1+\alpha \rho_{2} \phi+\alpha^{2} \rho_{5} \phi^{2}\right)^{2}},
$$

where $h_{2}$ is an integral constant and $a_{1}=(1 / 3) \alpha\left(2 c \rho_{2}-1-\right.$ $\left.2 \rho_{2}\right), a_{2}=(1 / 12) \alpha^{2}\left(-2 \rho_{1}-6 \rho_{5}+6 c \rho_{5}-3 \rho_{2}\right), a_{3}=-(1 / 30)$ $\alpha^{3}\left(6 \rho_{5}+4 \rho_{1} \rho_{2}+3 \rho_{4}\right), a_{4}=-(1 / 36) \alpha^{4}\left(4 \rho_{1} \rho_{5}+3 \rho_{4} \rho_{2}\right), a_{5}=$ $-(1 / 14) \rho_{4} \alpha^{5} \rho_{5}$.

(iii) When $m=3$, (12) can be reduced to

$$
\begin{aligned}
& y^{2}=\left(\phi ^ { 2 } \left[(c-1)+b_{1} \phi+b_{2} \phi^{2}+b_{3} \phi^{3}\right.\right. \\
&\left.\left.+b_{4} \phi^{4}+b_{5} \phi^{5}+b_{6} \phi^{6}+b_{7} \phi^{7}\right]+h_{3}\right) \\
& \times\left(\beta\left(1+\alpha \rho_{2} \phi+\alpha^{2} \rho_{5} \phi^{2}\right)^{3}\right)^{-1},
\end{aligned}
$$

where $h_{3}$ is an integral constant and $b_{1}=(1 / 3) \alpha\left(-1-4 \rho_{2}+\right.$ $\left.4 c \rho_{2}\right), b_{2}=(1 / 6) \alpha^{2}\left(-3 \rho_{2}-\rho_{1}+3 c \rho_{2}^{2}-3 \rho_{2}^{2}-6 \rho_{5}+6 c \rho_{5}\right)$, $b_{3}=(1 / 30) \alpha^{3}\left(-24 \rho_{2} \rho_{5}+24 c \rho_{2} \rho_{5}-3 \rho_{4}-12 \rho_{5}-6 \rho_{2}^{2}-8 \rho_{1} \rho_{2}\right)$, $b_{4}=(1 / 18) \alpha^{4}\left(-6 \rho_{5}^{2}-6 \rho_{2} \rho_{5}+6 c \rho_{5}^{2}-2 \rho_{2}^{2} \rho_{1}-3 \rho_{4} \rho_{2}-4 \rho_{1} \rho_{5}\right)$, $b_{5}=-(1 / 42) \alpha^{5}\left(8 \rho_{1} \rho_{2} \rho_{5}+6 \rho_{5}^{2}+6 \rho_{4} \rho_{5}+3 \rho_{4} \rho_{2}^{2}\right), b_{6}=$ $-(1 / 24) \alpha^{6} \rho_{5}\left(2 \rho_{1} \rho_{5}+3 \rho_{4} \rho_{2}\right), b_{7}=-(1 / 18) \rho_{4} \alpha^{7} \rho_{5}^{7}$. (iv) When $m$ is an integer and $m \geq 4$, (12) can be reduced to the following form:

$$
y^{2}=\frac{\phi^{2}\left[(c-1)+\gamma_{1} \phi+\gamma_{2} \phi^{2}+\cdots+\gamma_{2 m+1} \phi^{2 m+1}\right]+h_{m}}{\beta\left(1+\alpha \rho_{2} \phi+\alpha^{2} \rho_{5} \phi^{2}\right)^{m}},
$$

where $h_{m}(m \in \mathbf{N}$ and $m \geq 4)$ are integral constants and $\gamma_{1}, \gamma_{2}, \ldots, \gamma_{2 m+1}$ are certain expressions of the parameters $\rho_{1}$, $\rho_{2}, \rho_{4}, \rho_{5}, \alpha$, and $\beta$; these expressions are always determined from (12) once the $m$ is given by an idiographic integer; we omit them here because their expressions are tediously long.

\section{Exact Traveling Wave Solutions of (1) and Their Dynamic Properties}

In this section, we will investigate exact traveling wave solutions of (1) and discuss their dynamic properties under different kinds of parametric conditions in the greatly possible parameter regions; see the following discussions. For the convenience of discussion, we always consider the cases of all the integral constants as zero (i.e., $h_{i}=0, i=1,2, \ldots, m$ ) in the next discussions.

Case 1. Under the parametric conditions $h_{1}=0, m=1$, and $\rho_{5}=(1 / 4) \rho_{2}^{2},(13)$ can be reduced to

$$
y= \pm \frac{\phi \sqrt{(c-1)-(1 / 3) \alpha \phi-(1 / 6) \rho_{1} \alpha^{2} \phi^{2}-(1 / 10) \rho_{4} \alpha^{3} \phi^{3}}}{\sqrt{\beta}\left(1+(1 / 2) \alpha \rho_{2} \phi\right)} .
$$

(a) When $\rho_{4}<0, c=1$, and $\rho_{5}=(1 / 4) \rho_{2}^{2}$, substituting (17) into the first equation of (9) yields

$$
\frac{d \phi}{\sqrt{X_{11}(\phi)}}+\frac{1}{2} \alpha \rho_{2} \frac{d \phi}{\sqrt{X_{12}(\phi)}}= \pm \alpha \sqrt{-\frac{\alpha \rho_{4}}{10 \beta}} d \xi
$$

where $X_{11}(\phi)=\left(\phi-\phi_{1}\right)(\phi-0)^{3}\left(\phi-\phi_{2}\right)$ and $X_{12}(\phi)=(\phi-$ $\left.\phi_{1}\right)(\phi-0)\left(\phi-\phi_{2}\right)$ with $\phi_{1,2}=\left(5 \rho_{1} \pm \sqrt{25 \rho_{1}^{2}-120 \rho_{4}}\right) / 6\left(-\rho_{4}\right) \alpha$ and $\phi_{2}<0<\phi_{1}<\phi$. Taking $\phi(0)=\phi_{1}$ as the initial constant and then integrating (18), we obtain an implicit solution of (1) as follows:

$$
\begin{aligned}
& \frac{2 \sqrt{\phi_{1}-\phi_{2}}}{-\phi_{1} \phi_{2}} E\left(\theta_{1}, k_{1}\right)+\frac{2+\alpha \rho_{2} \phi_{2}}{\phi_{2} \sqrt{\phi_{1}-\phi_{2}}} F\left(\theta_{1}, k_{1}\right) \\
& =\alpha \sqrt{-\frac{\alpha \rho_{4}}{10 \beta}}|\xi|,
\end{aligned}
$$

where $F\left(\theta_{1}, k_{1}\right)$ is an incomplete elliptic integral of the first kind and $E\left(\theta_{1}, k_{1}\right)$ is a Legendre's incomplete elliptic integral of the second kind with $\theta_{1}=\arcsin \sqrt{\left(\eta-\phi_{1}\right) / \eta}, k_{1}=$ $\sqrt{-\phi_{2} /\left(\phi_{1}-\phi_{2}\right)}$, and $\eta(x, t)=\phi(\xi)$ with $\xi=x-t$.

(b) When $\rho_{4}>0, \rho_{1}>2 \sqrt{6 \rho_{4} / 5}, c=1$, and $\rho_{5}=(1 / 4) \rho_{2}^{2}$, substituting (17) into the first equation of (9) yields

$$
\frac{d \phi}{\sqrt{X_{21}(\phi)}}+\frac{1}{2} \alpha \rho_{2} \frac{d \phi}{\sqrt{X_{22}(\phi)}}= \pm \alpha \sqrt{\frac{\alpha \rho_{4}}{10 \beta}} d \xi
$$


where $X_{21}(\phi)=(0-\phi)^{3}\left(\phi-\widetilde{\phi}_{2}\right)\left(\phi-\widetilde{\phi}_{1}\right)$ and $X_{22}(\phi)=(0-$ $\phi)\left(\phi-\widetilde{\phi}_{2}\right)\left(\phi-\widetilde{\phi}_{1}\right)$ with $\widetilde{\phi}_{1,2}=\left(-5 \rho_{1} \mp 5 \sqrt{\rho_{1}^{2}-24 \rho_{4} / 5}\right) / 6 \rho_{4} \alpha$ and $\widetilde{\phi}_{1}<\widetilde{\phi}_{2}<\phi<0$. Taking $\phi(0)=\widetilde{\phi}_{2}$ as the initial constant and then integrating (20), we obtain an implicit solution of (1) as follows:

$$
\begin{aligned}
& -\frac{2 \widetilde{\phi}_{2}+\alpha \rho_{2}}{\widetilde{\phi}_{2} \sqrt{-\widetilde{\phi}_{1}}} F\left(\theta_{2}, k_{2}\right)+\frac{2}{\widetilde{\phi}_{2} \sqrt{-\widetilde{\phi}_{1}}} E\left(\theta_{2}, k_{2}\right) \\
& -\frac{2}{\widetilde{\phi}_{2}} \sqrt{\frac{\eta-\widetilde{\phi}_{2}}{\eta\left(\widetilde{\phi}_{1}-\eta\right)}}=\alpha \sqrt{\frac{\alpha \rho_{4}}{10 \beta}}|\xi|,
\end{aligned}
$$

where $\theta_{2}=\arcsin \sqrt{\widetilde{\phi}_{1}\left(\eta-\widetilde{\phi}_{2}\right) / \widetilde{\phi}_{2}\left(\eta-\widetilde{\phi}_{1}\right)}, k_{2}=\sqrt{\widetilde{\phi}_{2} / \widetilde{\phi}_{1}}$, and $\eta(x, t)=\phi(\xi)$ with $\xi=x-t$.

(c) When $\rho_{4}>0, \rho_{1}<-2 \sqrt{6 \rho_{4} / 5}, c=1$, and $\rho_{5}=(1 / 4) \rho_{2}^{2}$, substituting (17) into the first equation of (9) yields

$$
\frac{d \phi}{\sqrt{X_{31}(\phi)}}+\frac{1}{2} \alpha \rho_{2} \frac{d \phi}{\sqrt{X_{32}(\phi)}}= \pm \alpha \sqrt{\frac{\alpha \rho_{4}}{10 \beta}} d \xi,
$$

where $X_{31}(\phi)=\left(\widetilde{\phi}_{2}-\phi\right)\left(\phi-\widetilde{\phi}_{1}\right)(\phi-0)^{3}$ and $X_{32}(\phi)=\left(\widetilde{\phi}_{2}-\right.$ $\phi)\left(\phi-\widetilde{\phi}_{1}\right)(\phi-0)$ with $\widetilde{\phi}_{1,2}=\left(-5 \rho_{1} \mp 5 \sqrt{\rho_{1}^{2}-24 \rho_{4} / 5}\right) / 6 \rho_{4} \alpha$ and $0<\widetilde{\phi}_{1}<\phi<\widetilde{\phi}_{2}$. Taking $\phi(0)=\widetilde{\phi}_{1}$ as the initial constant and then integrating (22), we obtain an implicit solution of (1) as follows:

$$
\frac{2}{\widetilde{\phi}_{1} \sqrt{\widetilde{\phi}_{2}}} E\left(\theta_{2}, k_{2}\right)+\frac{\alpha \rho_{2}}{\sqrt{\widetilde{\phi}_{2}}} F\left(\theta_{2}, k_{2}\right)=\alpha \sqrt{\frac{\alpha \rho_{4}}{10 \beta}}|\xi|,
$$

where $\theta_{2}$ and $k_{2}$ are given above and $\eta(x, t)=\phi(\xi)$ with $\xi=$ $x-t$.

(d) When $\rho_{4}<0, c \neq 1$, and equation $(c-1)-(1 / 3) \alpha \phi-$ $(1 / 6) \rho_{1} \alpha^{2} \phi^{2}-(1 / 10) \rho_{4} \alpha^{3} \phi^{3}=0$ has three real roots, substituting (17) into the first equation of (9) yields

$$
\frac{d \phi}{\phi \sqrt{X_{4}(\phi)}}+\frac{1}{2} \alpha \rho_{2} \frac{d \phi}{\sqrt{X_{4}(\phi)}}= \pm \alpha \sqrt{-\frac{\alpha \rho_{4}}{10 \beta}} d \xi
$$

where $X_{4}(\phi)=\left(\phi-a_{1}\right)\left(\phi-b_{1}\right)\left(\phi-c_{1}\right)$ and the three real roots $a_{1}, b_{1}$, and $c_{1}$ are defined by $2 \sqrt{-p / 3} \cos (\tilde{\theta} / 3)-\left(5 \rho_{1} / 9 \alpha \rho_{4}\right)$, $2 \sqrt{-p / 3} \cos (\tilde{\theta} / 3+2 \pi / 3)-\left(5 \rho_{1} / 9 \alpha \rho_{4}\right), 2 \sqrt{-p / 3} \cos (\tilde{\theta} / 3+$ $4 \pi / 3)-\left(5 \rho_{1} / 9 \alpha \rho_{4}\right)$ with $p=-(5 / 27)\left(-18 \rho_{4}+5 \rho_{1}^{2}\right) /\left(\rho_{4}^{2} \alpha^{2}\right)<$ $0, q=-(10 / 729)\left(135 \rho_{1} \rho_{4}+729 c \rho_{4}^{2}-25 \rho_{1}^{3}-729 \rho_{4}^{2}\right) /\left(\rho_{4}^{3} \alpha^{3}\right)$, $\tilde{\theta}=\arccos \left[-(q / 2)\left(-p^{3} / 27\right)\right]^{-1 / 2}$. We always write $a_{1}>$ $b_{1}>c_{1}$; that is, the largest root is denoted by $a_{1}$ among the three real roots. Taking $\phi(0)=a_{1}$ as initial value and then integrating (24) once, we obtain a traveling wave solution as follows:

$$
\begin{aligned}
\frac{1}{\alpha_{1}^{2}} & {\left[F\left(\psi, k_{1}\right)-\left(\alpha_{1}^{2}-1\right) \Pi\left(\psi, \alpha_{1}^{2}, k_{1}\right)\right]+\frac{\alpha \rho_{2}}{\sqrt{a_{1}-c_{1}}} F\left(\psi, k_{1}\right) } \\
& =\alpha \sqrt{-\frac{\alpha \rho_{4}}{10 \beta}}|\xi|,
\end{aligned}
$$

where $\psi=\arcsin \sqrt{\left(\eta-a_{1}\right) /\left(\eta-b_{1}\right)}, \alpha_{1}=\sqrt{b_{1} / a_{1}}, k_{1}=$ $\sqrt{\left(b_{1}-c_{1}\right) /\left(a_{1}-c_{1}\right)}$, and $\eta(x, t)=\phi(\xi)$ with $\xi=x-c t$.

(e) When $\rho_{4}>0, c \neq 1$, and equation $(c-1)-(1 / 3) \alpha \phi-$ $(1 / 6) \rho_{1} \alpha^{2} \phi^{2}-(1 / 10) \rho_{4} \alpha^{3} \phi^{3}=0$ has three real roots $a_{1}, b_{1}$, and $c_{1}$, substituting (17) into the first equation of (9) yields

$$
\frac{d \phi}{\phi \sqrt{X_{5}(\phi)}}+\frac{1}{2} \alpha \rho_{2} \frac{d \phi}{\sqrt{X_{5}(\phi)}}= \pm \alpha \sqrt{\frac{\alpha \rho_{4}}{10 \beta}} d \xi,
$$

where $X_{5}(\phi)=\left(a_{1}-\phi\right)\left(\phi-b_{1}\right)\left(\phi-c_{1}\right)$ and $a_{1}, b_{1}$, and $c_{1}$ are given above. Taking $\phi(0)=a_{1}$ as initial value and then integrating (26) once, we obtain a traveling wave solution as follows:

$$
\frac{2}{a_{1} \sqrt{a_{1}-c_{1}}} \Pi\left(\varphi, \alpha_{2}^{2}, k_{2}\right)+\frac{\alpha \rho_{2}}{\sqrt{a_{1}-c_{1}}} F\left(\varphi, k_{2}\right)=\alpha \sqrt{\frac{\alpha \rho_{4}}{10 \beta}}|\xi|,
$$

where $\varphi=\arcsin \sqrt{\left(a_{1}-\eta\right) /\left(a_{1}-b_{1}\right)}, \alpha_{2}=\sqrt{\left(a_{1}-b_{1}\right) / a_{1}}$, $k_{2}=\sqrt{\left(a_{1}-b_{1}\right) /\left(a_{1}-c_{1}\right)}$, and $\eta(x, t)=\phi(\xi)$ with $\xi=x-c t$.

Case 2. Under the parametric conditions $m=1, h_{1}=0, \rho_{5}=$ $(1 / 4) \rho_{2}^{2}$, and $\rho_{4}=0,(13)$ can be reduced to

$$
y= \pm \frac{\phi \sqrt{(c-1)-(1 / 3) \alpha \phi-(1 / 6) \rho_{1} \alpha^{2} \phi^{2}}}{\sqrt{\beta}\left(1+(1 / 2) \alpha \rho_{2} \phi\right)} .
$$

Substituting (28) and $\rho_{5}=(1 / 4) \rho_{2}^{2}$ into the first equation of (9) yields

$$
\frac{d \phi}{\phi \sqrt{X_{6}(\phi)}}+\frac{1}{2} \alpha \rho_{2} \frac{d \phi}{\sqrt{X_{6}(\phi)}}= \pm \frac{1}{\sqrt{\beta}} d \xi
$$

where $X_{6}(\phi)=\widetilde{a}+\widetilde{b} \phi+\widetilde{c} \phi^{2}$ with $\widetilde{a}=(c-1), \widetilde{b}=-(1 / 3) \alpha$, and $\widetilde{c}=-(1 / 6) \rho_{1} \alpha^{2}$. Write $\Delta=4 \widetilde{a} \widetilde{c}-\widetilde{b}^{2}=(1 / 9) \alpha^{2}\left[6 \rho_{1}(c-1)+1\right]$.

(a) When $\tilde{a}>0, \tilde{c}>0, \Delta>0$ (i.e., $c>1,-(1 / 6(c-1))<$ $\rho_{1}<0$ ), integrating (29) and setting the integral constant as zero, we obtain two exact traveling wave solutions of implicit function type as follows:

$$
-\frac{1}{\sqrt{\widetilde{a}}} \operatorname{Arsh}\left(\frac{2 \widetilde{a}+\widetilde{b} \eta}{\eta \sqrt{\Delta}}\right)+\frac{\alpha \rho_{2}}{2 \sqrt{\widetilde{c}}} \operatorname{Arsh}\left(\frac{2 \widetilde{c} \eta+\widetilde{b}}{\sqrt{\Delta}}\right)= \pm \frac{\xi}{\sqrt{\beta}},
$$

where $\widetilde{a}, \widetilde{b}, \widetilde{c}$, and $\Delta$ are given above and $\eta(x, t)=\phi(\xi)$ with $\xi=x-c t$; the "Arsh" is an inverse function of the hyperbolicsine function, that is, $\sinh ^{-1}$.

(b) When $\widetilde{a}=0, \widetilde{c}<0, \Delta>0$ (i.e., $c=1, \rho_{1}>0$ ), integrating (29) and setting the integral constant as zero, we obtain two exact traveling wave solutions of implicit function type as follows:

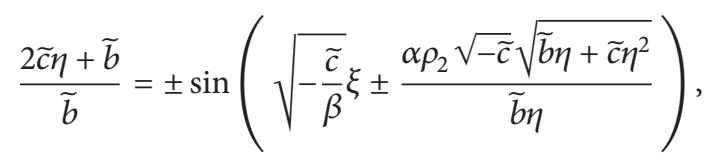

where $\eta(x, t)=\phi(\xi)$ with $\xi=x-t$. 
Case 3. Under the parametric conditions $m=1, h_{1}=0, c=$ $\left(\rho_{4} / 10 \rho_{2} \rho_{5}\right)+1-\left(1 / 3 \rho_{2}\right)$, and $\rho_{1}=\left(2 \rho_{5} / \rho_{2}\right)-\left(3 \rho_{4} / 5 \rho_{2}\right)+$ $\left(3 \rho_{2} \rho_{4} / 5 \rho_{5}\right),(13)$ can be reduced to

$$
y= \pm \phi \sqrt{\frac{3 \rho_{4}-10 \rho_{5}}{30 \beta \rho_{2} \rho_{5}}-\frac{\alpha \rho_{4}}{10 \beta \rho_{5}}} \phi .
$$

(a) When $\left(\left(3 \rho_{4}-10 \rho_{5}\right) / \rho_{2} \rho_{5}\right)>0$ and $\rho_{4} \neq 0$, substituting (32) into the first equation of (9) to integrate, we obtain a smooth solitary wave solution as follows:

$$
\eta(x, t) \equiv \phi(\xi)=-\frac{3 \rho_{4}-10 \rho_{5}}{3 \alpha \rho_{2} \rho_{4}} \operatorname{sech}^{2}\left(\frac{1}{2} \sqrt{\frac{3 \rho_{4}-10 \rho_{5}}{30 \beta \rho_{2} \rho_{5}}} \xi\right),
$$

where $\xi=x-\left[\left(\rho_{4} / 10 \rho_{2} \rho_{5}\right)+1-\left(1 / 3 \rho_{2}\right)\right] t$.

(b) When $\left(\left(3 \rho_{4}-10 \rho_{5}\right) / \rho_{2} \rho_{5}\right)<0$ and $\rho_{4} \neq 0$, substituting (32) into the first equation of (9) to integrate, we obtain a periodic wave solution as follows:

$$
\eta(x, t) \equiv \phi(\xi)=-\frac{3 \rho_{4}-10 \rho_{5}}{3 \alpha \rho_{2} \rho_{4}} \sec ^{2}\left(\frac{1}{2} \sqrt{\frac{10 \rho_{5}-3 \rho_{4}}{30 \beta \rho_{2} \rho_{5}}} \xi\right),
$$

where $\xi=x-\left[\left(\rho_{4} / 10 \rho_{2} \rho_{5}\right)+1-\left(1 / 3 \rho_{2}\right)\right] t$.

(c) When $\rho_{2}<0$ and $\rho_{4}=0$, (32) becomes

$$
y= \pm \frac{1}{\sqrt{-3 \beta \rho_{2}}} \phi, \quad(\phi>0) .
$$

Taking $\phi(0)=1$ as initial value, substituting (35) into the first equation of (9) to integrate, we obtain a peakon solution as follows:

$$
\eta(x, t)=e^{-\left(1 / \sqrt{-3 \beta \rho_{2}}\right)\left|x-\left(1-\left(1 / 3 \rho_{2}\right)\right) t\right|} .
$$

Case 4. Under the parametric conditions $m=2, h_{2}=0$, and $\rho_{4}=0,(14)$ can be reduced to

$$
y= \pm \frac{\phi \sqrt{(c-1)+a_{1} \phi+a_{2} \phi^{2}+a_{3} \phi^{3}+a_{4} \phi^{4}}}{\sqrt{\beta}\left(1+\alpha \rho_{2} \phi+\alpha^{2} \rho_{5} \phi^{2}\right)},
$$

where $a_{1}, a_{2}, a_{3}$, and $a_{4}$ are given above. Substituting (37) into the first equation of ( 9 ) yields

$$
\frac{d \phi}{\phi \sqrt{R_{1}(\phi)}}+\alpha \rho_{2} \frac{d \phi}{\sqrt{R_{1}(\phi)}}+\alpha^{2} \rho_{5} \frac{\phi d \phi}{\sqrt{R_{1}(\phi)}}= \pm \frac{d \xi}{\sqrt{\beta}}
$$

where $R_{1}(\phi)=(c-1)+a_{1} \phi+a_{2} \phi^{2}+a_{3} \phi^{3}+a_{4} \phi^{4}$. In fact, the cases of roots of the equation $R_{1}(\phi)=0$ determine the forms of solutions of (38); different kinds of cases of roots correspond to different kinds of solutions of (38). However, the expressions of the roots of the equation $R_{1}(\phi)=0$ are very complex, so we omit these expressions. Of course, the roots of the equation $R_{1}(\phi)=0$ can be solved once the parameters $\alpha$, $\rho_{1}, \rho_{2}$, and $\rho_{5}$ are fixed concretely. For example, we can obtain four real roots $2.919208358,-1.821216553,-3.236247427$, and -10.46174438 of the equation $R_{1}(\phi)=0$ when $\alpha=0.5$, $\rho_{1}=6.0, \rho_{2}=2.0, \rho_{5}=0.4$, and $c=4$ by using computer.
(1) When $\rho_{1} \rho_{5}>0$ and the equation $R_{1}(\phi)=0$ has four real roots $\phi_{1}, \phi_{2}, \phi_{3}$, and $\phi_{4}$, respectively, taking $\phi(0)=\phi_{1}, \phi_{2}$, $\phi_{3}, \phi_{4}$ as initial value and then integrating (38), we obtain four kinds of exact traveling wave solutions of implicit function type as follows:

$$
\begin{gathered}
-\Omega_{1} \Pi\left(\mu, \kappa_{1}, r\right)+\omega\left(\frac{1}{\phi_{4}}+\alpha \rho_{2}+\alpha^{2} \rho_{5} \phi_{4}\right) \mathrm{F}(\mu, r) \\
-\Omega_{2} \Pi\left(\mu, \kappa_{2}, r\right)=\frac{1}{3} \alpha^{2} \sqrt{\frac{\rho_{1} \rho_{5}}{\beta}}|\xi|, \\
\Omega_{3} \Pi\left(\lambda, \kappa_{3}, r\right)+\omega\left(\frac{1}{\phi_{3}}+\alpha \rho_{2}+\alpha^{2} \rho_{5} \phi_{3}\right) \mathrm{F}(\lambda, r) \\
+\Omega_{4} \Pi\left(\lambda, \kappa_{4}, r\right)=\frac{1}{3} \alpha^{2} \sqrt{\frac{\rho_{1} \rho_{5}}{\beta}}|\xi|, \\
\Omega_{1} \Pi\left(\gamma, \kappa_{5}, r\right)+\omega\left(\frac{1}{\phi_{2}}+\alpha \rho_{2}+\alpha^{2} \rho_{5} \phi_{2}\right) \mathrm{F}(\gamma, r) \\
+\Omega_{2} \Pi\left(\gamma, \kappa_{6}, r\right)=\frac{1}{3} \alpha^{2} \sqrt{\frac{\rho_{1} \rho_{5}}{\beta}}|\xi|, \\
-\Omega_{4} \Pi\left(\vartheta, \kappa_{8}, r\right)=\frac{1}{3} \alpha^{2} \sqrt{\frac{\rho_{1} \rho_{5}}{\beta}}|\xi|, \\
-\Omega_{3} \Pi\left(\vartheta, \kappa_{7}, r\right)+\omega\left(\frac{1}{\phi_{1}}+\alpha \rho_{2}+\alpha^{2} \rho_{5} \phi_{1}\right) \mathrm{F}(\vartheta, r)
\end{gathered}
$$

where $\omega=2 / \sqrt{\left(\phi_{1}-\phi_{3}\right)\left(\phi_{2}-\phi_{4}\right)}, \Omega_{1}=\omega\left(\phi_{1}-\phi_{4}\right) / \phi_{1} \phi_{4}$, $\Omega_{2}=\omega \alpha^{2} \rho_{5}\left(\phi_{4}-\phi_{1}\right), \Omega_{3}=\omega\left(\phi_{3}-\phi_{2}\right) / \phi_{2} \phi_{3}, \Omega_{4}=\omega \alpha^{2} \rho_{5}\left(\phi_{2}-\right.$ $\left.\phi_{3}\right), \mu=\arcsin \sqrt{\left(\phi_{2}-\phi_{4}\right)\left(\phi_{1}-\eta\right) /\left(\phi_{1}-\phi_{2}\right)\left(\eta-\phi_{4}\right)}$, and $\eta$ is limited by $\phi_{1} \geq \eta>\phi_{2}>\phi_{3}>\phi_{4}$; $\lambda=\arcsin \sqrt{\left(\phi_{1}-\phi_{3}\right)\left(\eta-\phi_{2}\right) /\left(\phi_{1}-\phi_{2}\right)\left(\eta-\phi_{4}\right)}$ and $\eta$ is limited by $\phi_{1} \geq \eta>\phi_{2}>\phi_{3}>\phi_{4} ; \gamma=$ $\arcsin \sqrt{\left(\phi_{2}-\phi_{4}\right)\left(\phi_{3}-\eta\right) /\left(\phi_{3}-\phi_{4}\right)\left(\phi_{2}-\eta\right)}$ and $\eta$ is limited by $\phi_{1}>\phi_{2}>\phi_{3}>\eta \geq \phi_{4} ; \vartheta=$ $\arcsin \sqrt{\left(\phi_{1}-\phi_{3}\right)\left(\eta-\phi_{4}\right) /\left(\phi_{3}-\phi_{4}\right)\left(\phi_{1}-\eta\right)}$ and $\eta$ is limited by $\phi_{1}>\phi_{2}>\phi_{3} \geq \eta>\phi_{4}$, and $\kappa_{1}=\phi_{4}\left(\phi_{2}-\phi_{1}\right) / \phi_{1}\left(\phi_{2}-\phi_{4}\right)$, $\kappa_{2}=\left(\phi_{2}-\phi_{1}\right) /\left(\phi_{2}-\phi_{4}\right), \kappa_{3}=\phi_{3}\left(\phi_{1}-\phi_{2}\right) / \phi_{2}\left(\phi_{1}-\phi_{3}\right), \kappa_{4}=\left(\phi_{1}-\right.$ $\left.\phi_{2}\right) /\left(\phi_{1}-\phi_{3}\right), \kappa_{5}=\phi_{2}\left(\phi_{3}-\phi_{4}\right) / \phi_{3}\left(\phi_{2}-\phi_{4}\right), \kappa_{6}=\left(\phi_{3}-\phi_{4}\right) /\left(\phi_{2}-\right.$ $\left.\phi_{4}\right), \kappa_{7}=\phi_{1}\left(\phi_{4}-\phi_{3}\right) / \phi_{4}\left(\phi_{1}-\phi_{3}\right), \kappa_{8}=\left(\phi_{4}-\phi_{3}\right) /\left(\phi_{1}-\phi_{3}\right)$, and $r=\sqrt{\left(\phi_{1}-\phi_{2}\right)\left(\phi_{3}-\phi_{4}\right) /\left(\phi_{1}-\phi_{3}\right)\left(\phi_{2}-\phi_{4}\right)}$.

(2) When $\rho_{1} \rho_{5}<0$ and the equation $R_{1}(\phi)=0$ has four real roots $\varphi_{1}, \varphi_{2}, \varphi_{3}$, and $\varphi_{4}$, respectively, taking $\phi(0)=$ $\varphi_{1}, \varphi_{2}, \varphi_{3}, \varphi_{4}$ as initial value and then integrating (38), we obtain another four kinds of exact traveling wave solutions of implicit function type as follows:

$$
\begin{gathered}
Q_{1} \Pi\left(\nu, m_{1}, q\right)+\omega\left(\frac{1}{\varphi_{2}}+\alpha \rho_{2}+\alpha^{2} \rho_{5} \varphi_{2}\right) \mathrm{F}(\nu, q) \\
+Q_{2} \Pi\left(\nu, m_{2}, q\right)=\frac{1}{3} \alpha^{2} \sqrt{-\frac{\rho_{1} \rho_{5}}{\beta}}|\xi|,
\end{gathered}
$$




$$
\begin{gathered}
-Q_{1} \Pi\left(\epsilon, m_{3}, q\right)+\omega\left(\frac{1}{\varphi_{1}}+\alpha \rho_{2}+\alpha^{2} \rho_{5} \varphi_{1}\right) \mathrm{F}(\epsilon, q) \\
-Q_{2} \Pi\left(\epsilon, m_{4}, q\right)=\frac{1}{3} \alpha^{2} \sqrt{-\frac{\rho_{1} \rho_{5}}{\beta}}|\xi| \\
Q_{3} \Pi\left(\delta, m_{5}, q\right)+\omega\left(\frac{1}{\varphi_{4}}+\alpha \rho_{2}+\alpha^{2} \rho_{5} \varphi_{4}\right) \mathrm{F}(\delta, q) \\
+Q_{4} \Pi\left(\delta, m_{6}, q\right)=\frac{1}{3} \alpha^{2} \sqrt{-\frac{\rho_{1} \rho_{5}}{\beta}}|\xi|, \\
-Q_{3} \Pi\left(\zeta, m_{7}, q\right)+\omega\left(\frac{1}{\varphi_{3}}+\alpha \rho_{2}+\alpha^{2} \rho_{5} \varphi_{3}\right) \mathrm{F}(\zeta, q) \\
-Q_{4} \Pi\left(\zeta, m_{8}, q\right)=\frac{1}{3} \alpha^{2} \sqrt{-\frac{\rho_{1} \rho_{5}}{\beta}}|\xi|,
\end{gathered}
$$

where $Q_{1}=\omega\left(\varphi_{2}-\varphi_{1}\right) / \varphi_{1} \varphi_{2}, Q_{2}=\omega \alpha^{2} \rho_{5}\left(\varphi_{1}-\varphi_{2}\right)$, $Q_{3}=\omega\left(\varphi_{4}-\varphi_{3}\right) / \varphi_{3} \varphi_{4}, Q_{4}=\omega \alpha^{2} \rho_{5}\left(\varphi_{3}-\varphi_{4}\right)$, $\nu=\arcsin \sqrt{\left(\varphi_{2}-\varphi_{4}\right)\left(\eta-\varphi_{1}\right) /\left(\varphi_{1}-\varphi_{4}\right)\left(\eta-\varphi_{2}\right)}$, and $\eta$ is defined by $\varphi_{1} \geq \eta>\varphi_{2}>\varphi_{3}>\varphi_{4}$; $\epsilon=\arcsin \sqrt{\left(\varphi_{1}-\varphi_{3}\right)\left(\varphi_{2}-\eta\right) /\left(\varphi_{2}-\varphi_{3}\right)\left(\varphi_{1}-\eta\right)}$ and $\eta$ is defined by $\varphi_{1}>\varphi_{2}>\eta \geq \varphi_{3}>\varphi_{4}$; $\delta=\arcsin \sqrt{\left(\varphi_{2}-\varphi_{4}\right)\left(\eta-\varphi_{3}\right) /\left(\varphi_{2}-\varphi_{3}\right)\left(\eta-\varphi_{4}\right)}$ and $\eta$ is defined $\operatorname{by} \varphi_{1}>\varphi_{2} \geq \eta>\varphi_{3}>\varphi_{4} ; \zeta=$ $\arcsin \sqrt{\left(\varphi_{1}-\varphi_{3}\right)\left(\varphi_{4}-\eta\right) /\left(\varphi_{1}-\varphi_{4}\right)\left(\varphi_{3}-\eta\right)}$ and $\eta$ is defined by $\varphi_{1}>\varphi_{2}>\varphi_{3}>\varphi_{4}>\eta$, and $m_{1}=\varphi_{2}\left(\varphi_{1}-\right.$ $\left.\varphi_{4}\right) / \varphi_{1}\left(\varphi_{2}-\varphi_{4}\right), m_{2}=\left(\varphi_{1}-\varphi_{4}\right) /\left(\varphi_{2}-\varphi_{4}\right), m_{3}=\varphi_{1}\left(\varphi_{2}-\right.$ $\left.\varphi_{3}\right) / \varphi_{2}\left(\varphi_{1}-\varphi_{3}\right), m_{4}=\left(\varphi_{2}-\varphi_{3}\right) /\left(\varphi_{1}-\varphi_{3}\right), m_{5}=\varphi_{4}\left(\varphi_{2}-\right.$ $\left.\varphi_{3}\right) / \varphi_{3}\left(\varphi_{2}-\varphi_{4}\right), m_{6}=\left(\varphi_{2}-\varphi_{3}\right) /\left(\varphi_{2}-\varphi_{4}\right), m_{7}=\varphi_{3}\left(\varphi_{1}-\right.$ $\left.\varphi_{4}\right) / \varphi_{4}\left(\varphi_{1}-\varphi_{3}\right), m_{8}=\left(\varphi_{1}-\varphi_{4}\right) /\left(\varphi_{1}-\varphi_{3}\right)$, and $q=$ $\sqrt{\left(\varphi_{2}-\varphi_{3}\right)\left(\varphi_{1}-\varphi_{4}\right) /\left(\varphi_{1}-\varphi_{3}\right)\left(\varphi_{2}-\varphi_{4}\right)}$.

Case 5. Under the parametric conditions $m=2, h_{2}=0, c=$ $\left(3 \rho_{4} / 56 \rho_{2} \rho_{5}\right)+1-\left(1 / 4 \rho_{2}\right)$, and $\rho_{1}=\left(9 \rho_{5} / 4 \rho_{2}\right)-\left(27 \rho_{4} / 56 \rho_{2}\right)+$ $\left(15 \rho_{2} \rho_{4} / 28 \rho_{5}\right),(14)$ can be reduced to

$$
y= \pm \phi \sqrt{\frac{3 \rho_{4}-14 \rho_{5}}{56 \beta \rho_{2} \rho_{5}}-\frac{\alpha \rho_{4}}{14 \beta \rho_{5}}} \phi .
$$

(a) When $\left(3 \rho_{4}-14 \rho_{5}\right) / \rho_{2} \rho_{5}>0$ and $\rho_{4} \neq 0$, substituting (44) into the first equation of (9) to integrate, we obtain a smooth solitary wave solution as follows:

$$
\eta(x, t) \equiv \phi(\xi)=-\frac{3 \rho_{4}-14 \rho_{5}}{4 \alpha \rho_{2} \rho_{4}} \operatorname{sech}^{2}\left(\frac{1}{2} \sqrt{\frac{3 \rho_{4}-14 \rho_{5}}{56 \beta \rho_{2} \rho_{5}} \xi}\right)
$$

where $\xi=x-\left[\left(3 \rho_{4} / 56 \rho_{2} \rho_{5}\right)+1-\left(1 / 4 \rho_{2}\right)\right]$ t. (b) When $\left(3 \rho_{4}-14 \rho_{5} / \rho_{2} \rho_{5}\right)<0$ and $\rho_{4} \neq 0$, substituting (44) into the first equation of (9) to integrate, we obtain a periodic wave solution as follows:

$$
\eta(x, t) \equiv \phi(\xi)=-\frac{3 \rho_{4}-14 \rho_{5}}{4 \alpha \rho_{2} \rho_{4}} \sec ^{2}\left(\frac{1}{2} \sqrt{\frac{14 \rho_{5}-3 \rho_{4}}{56 \beta \rho_{2} \rho_{5}}} \xi\right),
$$

where $\xi=x-\left[\left(3 \rho_{4} / 56 \rho_{2} \rho_{5}\right)+1-\left(1 / 4 \rho_{2}\right)\right] t$.

(c) When $\rho_{2}<0$ and $\rho_{4}=0$, (44) becomes

$$
y= \pm \frac{1}{2 \sqrt{-\beta \rho_{2}}} \phi, \quad(\phi>0) .
$$

Taking $\phi(0)=1$ as initial value, substituting (47) into the first equation of (9) to integrate, we obtain a peakon solution as follows:

$$
\eta(x, t)=e^{-\left(1 / 2 \sqrt{-\beta \rho_{2}}\right)\left|x-\left(1-\left(1 / 4 \rho_{2}\right)\right) t\right|} .
$$

Case 6. Under the parametric conditions $m=3, h_{3}=0, c=$ $\left(\rho_{4} / 30 \rho_{2} \rho_{5}\right)+1-\left(1 / 5 \rho_{2}\right)$, and $\rho_{1}=\left(12 \rho_{5} / 5 \rho_{2}\right)-\left(2 \rho_{4} / 5 \rho_{2}\right)+$ $\left(\rho_{2} \rho_{4} / 2 \rho_{5}\right),(15)$ can be reduced to

$$
y= \pm \phi \sqrt{\frac{3 \rho_{4}-18 \rho_{5}}{90 \beta \rho_{2} \rho_{5}}-\frac{\alpha \rho_{4}}{18 \beta \rho_{5}} \phi} .
$$

(a) When $\left(\left(3 \rho_{4}-18 \rho_{5}\right) / \rho_{2} \rho_{5}\right)>0$ and $\rho_{4} \neq 0$, substituting (49) into the first equation of (9) to integrate, we obtain a smooth solitary wave solution as follows:

$$
\eta(x, t) \equiv \phi(\xi)=-\frac{3 \rho_{4}-18 \rho_{5}}{5 \alpha \rho_{2} \rho_{4}} \operatorname{sech}^{2}\left(\frac{1}{2} \sqrt{\frac{3 \rho_{4}-18 \rho_{5}}{90 \beta \rho_{2} \rho_{5}}} \xi\right),
$$

where $\xi=x-\left[\left(\rho_{4} / 30 \rho_{2} \rho_{5}\right)+1-\left(1 / 5 \rho_{2}\right)\right] t$.

(b) When $\left(\left(3 \rho_{4}-18 \rho_{5}\right) / \rho_{2} \rho_{5}\right)<0$ and $\rho_{4} \neq 0$, substituting (49) into the first equation of (9) to integrate, we obtain a periodic wave solution as follows:

$$
\eta(x, t) \equiv \phi(\xi)=-\frac{3 \rho_{4}-18 \rho_{5}}{5 \alpha \rho_{2} \rho_{4}} \sec ^{2}\left(\frac{1}{2} \sqrt{\frac{18 \rho_{5}-3 \rho_{4}}{90 \beta \rho_{2} \rho_{5}}} \xi\right),
$$

where $\xi=x-\left[\left(\rho_{4} / 30 \rho_{2} \rho_{5}\right)+1-\left(1 / 5 \rho_{2}\right)\right] t$.

(c) When $\rho_{2}<0$ and $\rho_{4}=0$, (49) becomes

$$
y= \pm \frac{1}{\sqrt{-5 \beta \rho_{2}}} \phi, \quad(\phi>0) .
$$

Taking $\phi(0)=1$ as initial value, substituting (52) into the first equation of (9) to integrate, we obtain a peakon solution as follows:

$$
\eta(x, t)=e^{-\left(1 / \sqrt{-5 \beta \rho_{2}}\right)\left|x-\left(1-\left(1 / 5 \rho_{2}\right)\right) t\right|} .
$$

Case 7. Under the parametric conditions $m=4, h_{4}=0, c=$ $\left(3 \rho_{4} / 132 \rho_{2} \rho_{5}\right)+1-\left(1 / 6 \rho_{2}\right)$, and $\rho_{1}=\left(5 \rho_{5} / 2 \rho_{2}\right)-\left(15 \rho_{4} / 44 \rho_{2}\right)+$ $\left(21 \rho_{2} \rho_{4} / 44 \rho_{5}\right),(16)$ can be reduced to

$$
y= \pm \phi \sqrt{\frac{3 \rho_{4}-22 \rho_{5}}{132 \beta \rho_{2} \rho_{5}}-\frac{\alpha \rho_{4}}{22 \beta \rho_{5}} \phi} .
$$




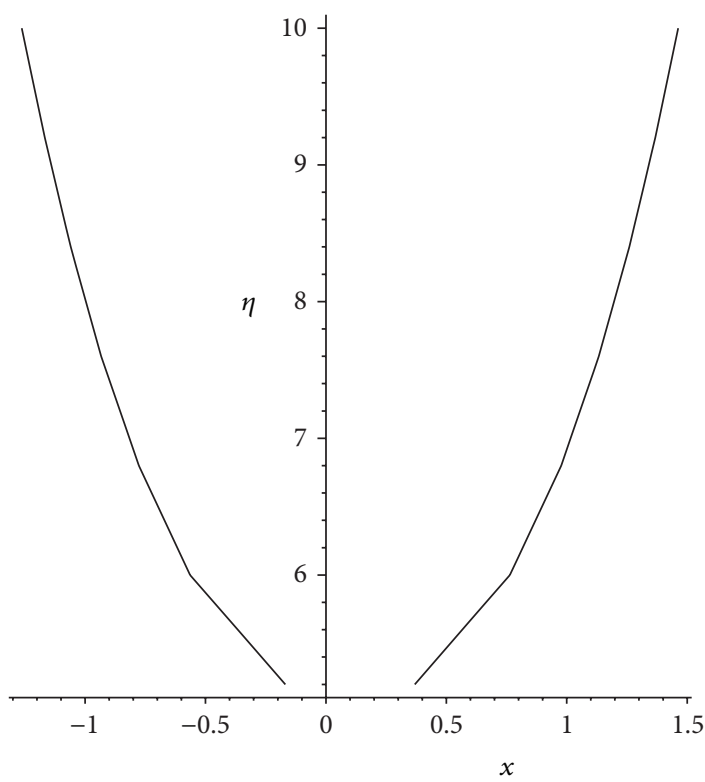

(a) Dark broken-soliton

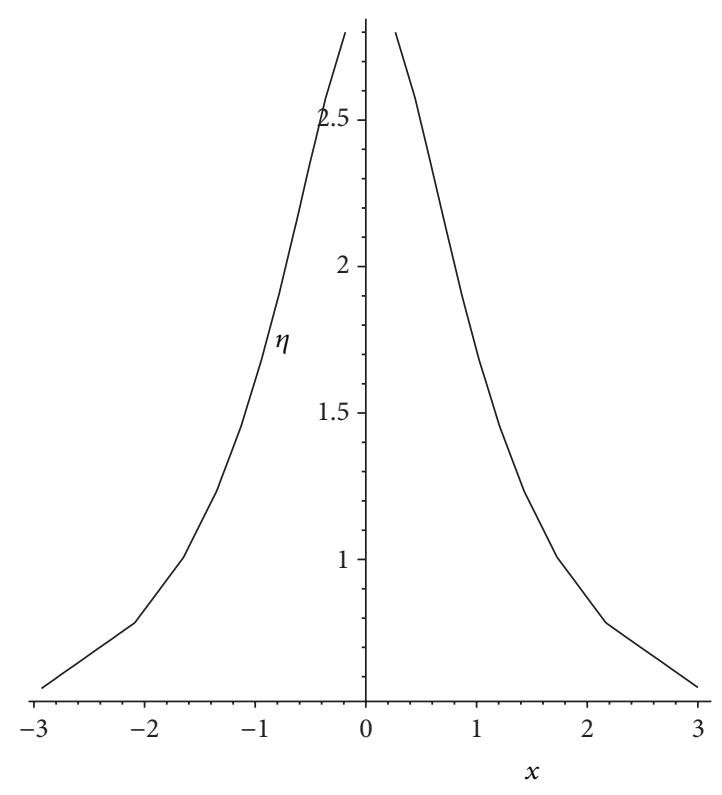

(b) Bright broken-soliton

FIGURE 1: The graphs of solutions (19) and (39) under some fixed parametric values.

(a) When $\left(\left(3 \rho_{4}-22 \rho_{5}\right) / \rho_{2} \rho_{5}\right)>0$ and $\rho_{4} \neq 0$, substituting (54) into the first equation of (9) to integrate, we obtain a smooth solitary wave solution as follows:

$$
\eta(x, t) \equiv \phi(\xi)=-\frac{3 \rho_{4}-22 \rho_{5}}{6 \alpha \rho_{2} \rho_{4}} \operatorname{sech}^{2}\left(\frac{1}{2} \sqrt{\frac{3 \rho_{4}-22 \rho_{5}}{132 \beta \rho_{2} \rho_{5}}} \xi\right),
$$

where $\xi=x-\left[\left(3 \rho_{4} / 132 \rho_{2} \rho_{5}\right)+1-\left(1 / 6 \rho_{2}\right)\right] t$.

(b) When $\left(\left(3 \rho_{4}-22 \rho_{5}\right) / \rho_{2} \rho_{5}\right)<0$ and $\rho_{4} \neq 0$, substituting (54) into the first equation of (9) to integrate, we obtain a periodic wave solution as follows:

$$
\eta(x, t) \equiv \phi(\xi)=-\frac{3 \rho_{4}-22 \rho_{5}}{6 \alpha \rho_{2} \rho_{4}} \sec ^{2}\left(\frac{1}{2} \sqrt{\frac{22 \rho_{5}-3 \rho_{4}}{132 \beta \rho_{2} \rho_{5}}} \xi\right),
$$

where $\xi=x-\left[\left(3 \rho_{4} / 132 \rho_{2} \rho_{5}\right)+1-\left(1 / 6 \rho_{2}\right)\right] t$.

(c) When $\rho_{2}<0$ and $\rho_{4}=0$, (54) becomes

$$
y= \pm \frac{1}{\sqrt{-6 \beta \rho_{2}}} \phi, \quad(\phi>0) .
$$

Taking $\phi(0)=1$ as initial value, substituting (57) into the first equation of (9) to integrate, we obtain a peakon solution as follows:

$$
\eta(x, t)=e^{-\left(1 / \sqrt{-6 \beta \rho_{2}}\right)\left|x-\left(1-\left(1 / 6 \rho_{2}\right)\right) t\right|} .
$$

When the value of $m$ goes on increasing, the expression (16) becomes more and more complex; thus we cannot obtain exact solutions of (1) as in Cases 1, 2, and 4 under general parameter conditions by integrating this expression. But the exact smooth solitary wave solutions and nonsmooth peakon solutions can always be obtained under certain special parameter conditions. From Cases 3, 6, and 7, by using the mathematical induction, we easily obtain the following results.

Under the parametric conditions $h_{m}=0, c=\left(3 \rho_{4} /(2 m+\right.$ 3) $\left.(2 m+4) \rho_{2} \rho_{5}\right)+1-\left(1 /(m+2) \rho_{2}\right)$, and $\rho_{1}=\left(3(m+1) \rho_{5} /(m+\right.$ 2) $\left.\rho_{2}\right)-\left(9(m+1) \rho_{4} /(2 m+3)(2 m+4) \rho_{2}\right)+\left(3(m+3) \rho_{2} \rho_{4} / 4(2 m+\right.$ 3) $\rho_{5}$ ) and all-in positive integers $m$, (16) can be reduced to

$$
y= \pm \phi \sqrt{\frac{3 \rho_{4}-(4 m+6) \rho_{5}}{(2 m+3)(2 m+4) \beta \rho_{2} \rho_{5}}-\frac{\alpha \rho_{4}}{(4 m+6) \beta \rho_{5}} \phi .}
$$

(a) When $\left(3 \rho_{4}-(4 m+6) \rho_{5} / \rho_{2} \rho_{5}\right)>0$ and $\rho_{4} \neq 0$, substituting (59) into the first equation of (9) to integrate, for all-in positive integers $m$, we obtain a series of smooth solitary wave solutions as follows:

$$
\begin{aligned}
\eta(x, t) \equiv & \phi(\xi)=-\frac{3 \rho_{4}-(4 m+6) \rho_{5}}{(m+2) \alpha \rho_{2} \rho_{4}} \\
& \times \operatorname{sech}^{2}\left(\frac{1}{2} \sqrt{\frac{3 \rho_{4}-(4 m+6) \rho_{5}}{(2 m+3)(2 m+4) \beta \rho_{2} \rho_{5}}} \xi\right),
\end{aligned}
$$

where $\xi=x-\left[\left(3 \rho_{4} /(2 m+3)(2 m+4) \rho_{2} \rho_{5}\right)+1-\left(1 /(m+2) \rho_{2}\right)\right] t$. Obviously, when $m=1,2,3,4$, the solution (60), respectively, becomes the solutions (33), (45), (50), and (55).

(b) When $\left(\left(3 \rho_{4}-(4 m+6) \rho_{5}\right) / \rho_{2} \rho_{5}\right)<0$ and $\rho_{4} \neq 0$, substituting (59) into the first equation of (9) to integrate, for 


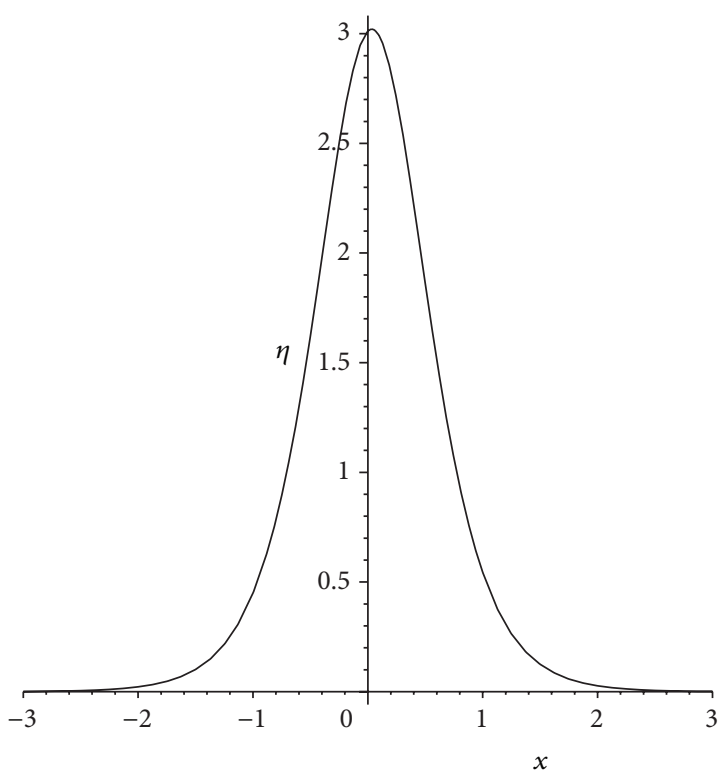

(a) $m=1$

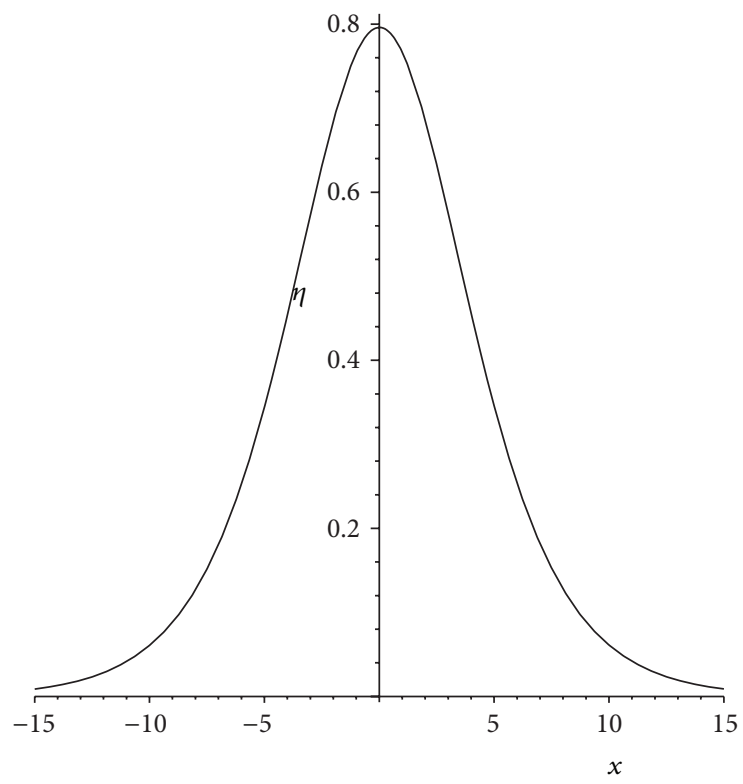

(c) $m=40$

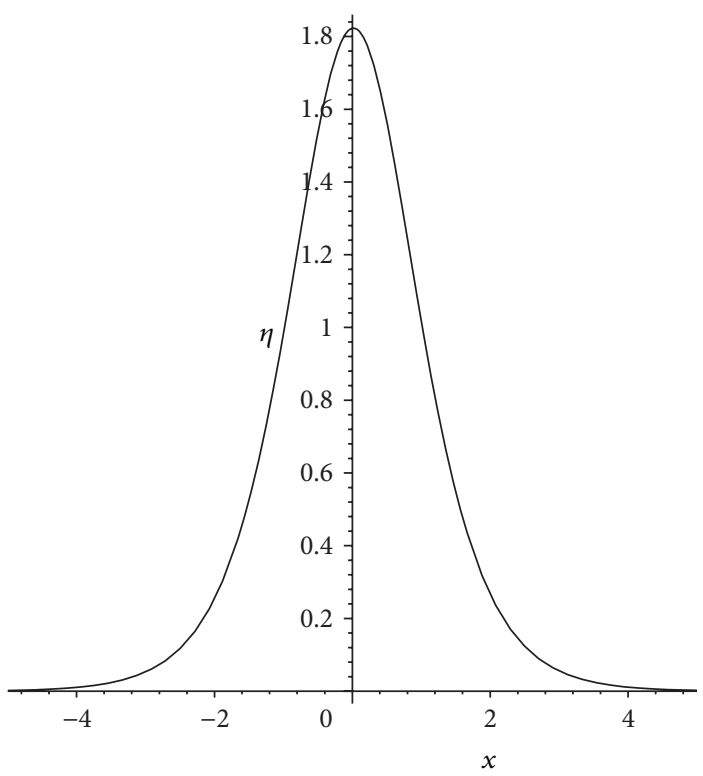

(b) $m=4$

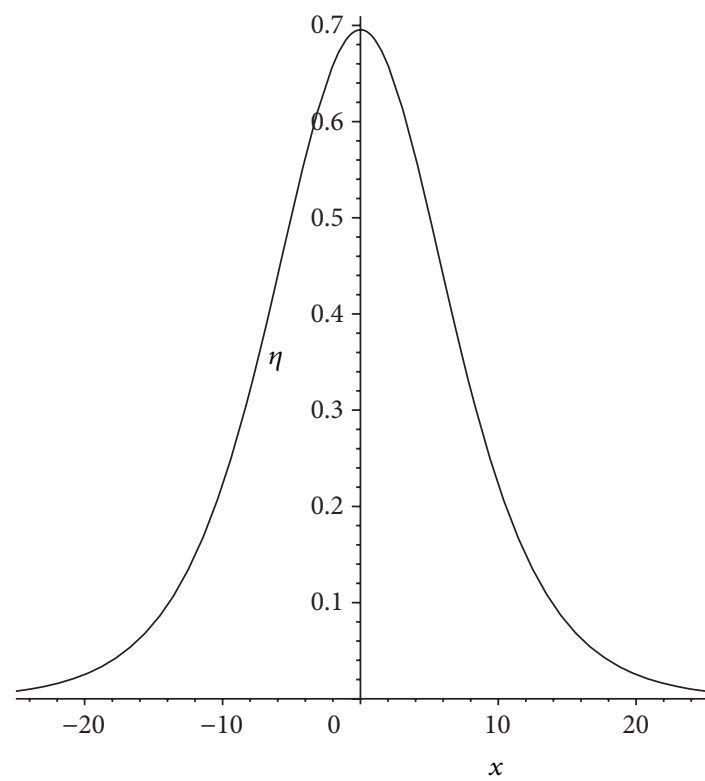

(d) $m=100$

FIGURE 2: The graphs of solution (60) under some fixed parametric values.

all-in positive integers $m$, we obtain a series of periodic wave solutions as follows:

$$
\begin{aligned}
\eta(x, t) \equiv & \phi(\xi)=-\frac{3 \rho_{4}-(4 m+6) \rho_{5}}{(m+2) \alpha \rho_{2} \rho_{4}} \\
& \times \sec ^{2}\left(\frac{1}{2} \sqrt{\frac{(4 m+6) \rho_{5}-3 \rho_{4}}{(2 m+3)(2 m+4) \beta \rho_{2} \rho_{5}}} \xi\right),
\end{aligned}
$$

where $\xi=x-\left[\left(3 \rho_{4} /(2 m+3)(2 m+4) \rho_{2} \rho_{5}\right)+1-(1 /(m+\right.$ 2) $\left.\left.\rho_{2}\right)\right] t$. In particular, when $m=1,2,3,4$, the solution (61), respectively, becomes the solutions (34), (46), (51), and (56). (c) When $\rho_{2}<0$ and $\rho_{4}=0$, (59) becomes

$$
y= \pm \frac{1}{-(2 m+3)(2 m+4) \beta \rho_{2}} \phi, \quad(\phi>0) .
$$

Taking $\phi(0)=1$ as initial value, substituting (62) into the first equation of (9) to integrate, for all-in positive integers $m$, we obtain a series of peakon solutions as follows:

$$
\eta(x, t)=e^{-\left(1 / \sqrt{-(m+2) \beta \rho_{2}}\right)\left|x-\left(1-\left(1 /(m+2) \rho_{2}\right)\right) t\right|} .
$$

Similarly, when $m=1,2,3,4$, the solution (63), respectively, becomes the solutions (36), (48), (53), and (58). 


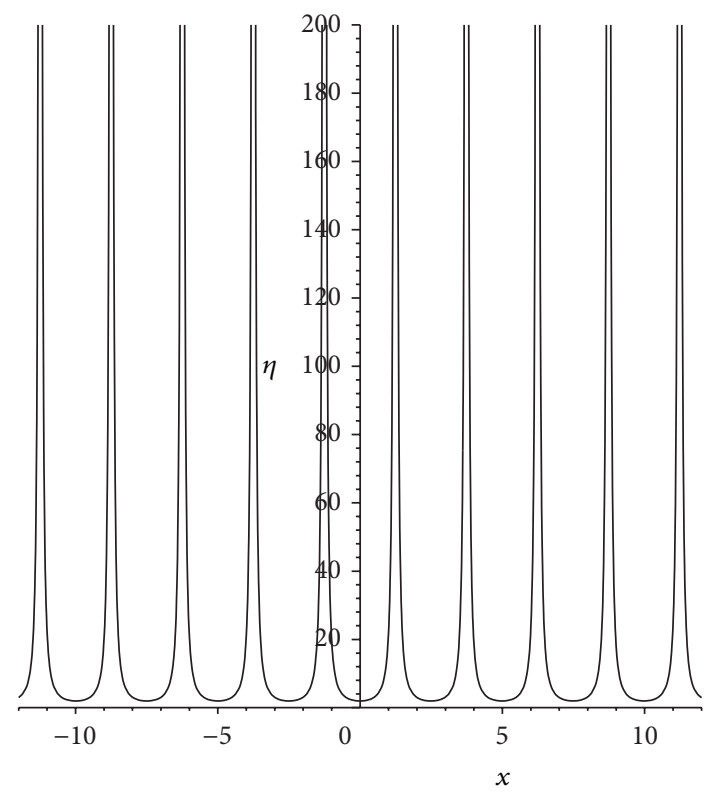

(a) $m=1$

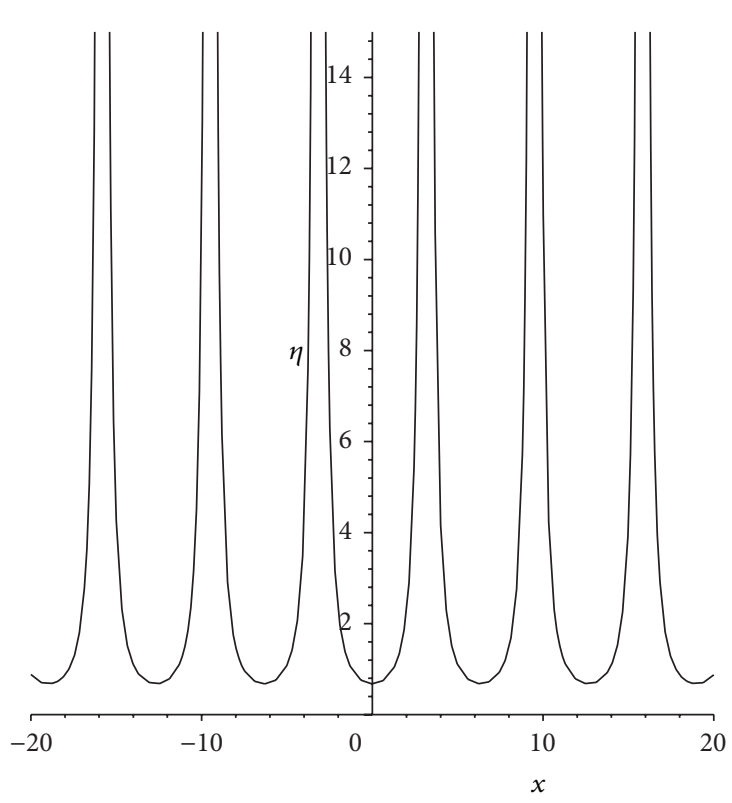

(b) $m=4$

FIGURE 3: The graphs of solution (61) under some fixed parametric values.

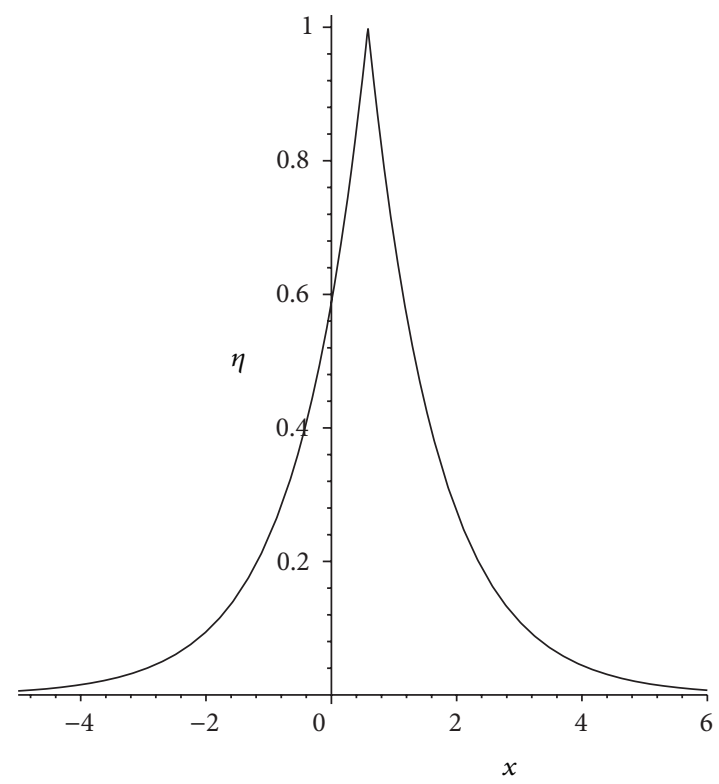

(a) $m=1$

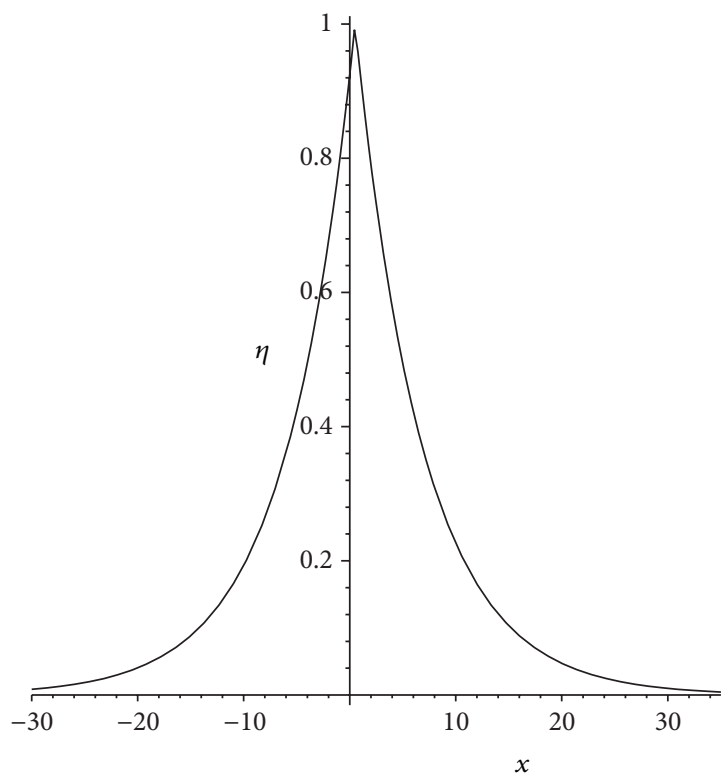

(b) $m=100$

FIgURE 4: The graphs of solution (63) under some fixed parametric values.

In order to intuitively describe and expediently discuss the dynamic properties of the above exact traveling wave solutions of implicit type and explicit type, we draw their profile graphs; see Figures 1, 2, 3, and 4 and the discussions below them.

When $\alpha=0.5, \beta=0.25, \rho_{1}=3, \rho_{2}=2, \rho_{4}=-2.5, t=0.1$, $x \in[-3,3]$, and $\eta \in[0,10]$, we draw graph of solution (19); see Figure 1(a). When $\alpha=0.5, \beta=0.25, \rho_{1}=6, \rho_{2}=2$, $\rho_{5}=0.4, c=4, t=0.01, x \in[-3,3]$, and $\eta \in[0,2.8]$, we draw profile graph of solution (39); see Figure 1(b).

Figure 1(a) shows a shape of dark broken-soliton; it seems that a dark soliton is broken. Figure 1(b) shows a shape of bright broken-soliton; it seems that a bright soliton is broken.

When $\alpha=0.5, \beta=0.25, \rho_{2}=-0.8, \rho_{4}=8, \rho_{5}=-0.5$, $t=0.01$, and $m$ is taking different values, we draw graphs of solution (60); see Figures 2(a)-2(d). 
Figure 2 shows four smooth solitary waves; it is easy to find that their amplitudes and width of waves are different; the amplitude and width of wave decrease as the value of $m$ increases under the same parametric conditions.

When $\alpha=0.5, \beta=0.25, \rho_{2}=-0.8, \rho_{4}=8, \rho_{5}=0.5$, $t=0.01$, and $m$ is taking different values, we draw graphs of solution (61); see Figures 3(a) and 3(b).

Figure 3 shows two periodic waves of blow-up type; it is easy to find that their width of waves is also different; the width of periodic wave increases as the value of $m$ increases under the same parametric conditions.

When $\beta=0.2, \rho_{2}=-2, t=0.5$, and $m$ is taking different values, we draw graphs of solution (63); see Figures 4(a) and 4(b).

Figure 4 shows two peakon waves; it is easy to find that their amplitudes are the same, but the width of waves and velocities of waves are different; the width of wave increases as the value of $m$ increases; the velocities of waves decrease as the value of $m$ increases under the same parametric condition.

\section{Conclusion}

In this work, by using the integral bifurcation method together with factoring technique, we investigate exact traveling wave solutions of (1) within more extensive parameter ranges. Some exact traveling wave solutions such as brokensoliton solutions (19), (21), (23), (25) (27), (39)-(42), and (43), periodic wave solutions of blow-up type (34), (46), (51), (56), and (61), smooth solitary wave solutions (33), (45), (50), (55), and (60), and nonsmooth peakon solutions (35), (48), (53), (58), and (63) are obtained. It is very worthy to mention solutions (60) and (63); solution (60) denotes a series of smooth solitary waves; solution (63) denotes a series of nonsmooth peakon waves according as the positive integer $m$ increase. Compared with the literature [9], exact solutions obtained in this paper are much more than those in [9]; only one exact solitary wave solution is obtained in [9]. Indeed, we enrich the results of exact solutions based on the works in $[8,9]$.

In addition, the results obtained in this work are very different from those in existing literatures. However, the exact solutions which we obtained in this paper are not much yet because the model studied in this work is very complex equation. Therefore, we hope more and more researchers pay attention to investigations for exact solutions of (1) in future work.

\section{Conflict of Interests}

The author declares that there is no conflict of interests regarding the publication of this paper.

\section{Acknowledgments}

This work is supported by the Natural Science Foundation of China under Grant no. 11361023, the Natural Science Foundation of Chongqing Normal University under Grant no. 13XLR20, and the Program Foundation of Chongqing
Innovation Team Project in University under Grant no. KJTD201308.

\section{References}

[1] A. S. Fokas, "On a class of physically important integrable equations," Physica D, vol. 87, no. 1-4, pp. 145-150, 1995.

[2] E. Tzirtzilakis, V. Marinakis, C. Apokis, and T. Bountis, "Soliton-like solutions of higher order wave equations of the Korteweg-de Vries type," Journal of Mathematical Physics, vol. 43, no. 12, pp. 6151-6165, 2002.

[3] E. Tzirtzilakis, M. Xenos, V. Marinakis, and T. C. Bountis, "Interactions and stability of solitary waves in shallow water," Chaos, Solitons \& Fractals, vol. 14, no. 1, pp. 87-95, 2002.

[4] J. Li, W. Rui, Y. Long, and B. He, “Travelling wave solutions for higher-order wave equations of KdV type. III," Mathematical Biosciences and Engineering, vol. 3, no. 1, pp. 125-135, 2006.

[5] W. Rui, Y. Long, and B. He, "Some new travelling wave solutions with singular or nonsingular character for the higher order wave equation of KdV type (III)," Nonlinear Analysis: Theory, Methods \& Applications, vol. 70, no. 11, pp. 3816-3828, 2009.

[6] B. Fuchssteiner and A. S. Fokas, "Symplectic structures, their Bäcklund transformations and hereditary symmetries," Physica D, vol. 4, no. 1, pp. 47-66, 1981/82.

[7] R. Camassa and D. D. Holm, "An integrable shallow water equation with peaked solitons," Physical Review Letters, vol. 71, no. 11, pp. 1661-1664, 1993.

[8] X. Wu, W. Rui, and X. Hong, "A generalized KdV equation of neglecting the highest-order infinitesimal term and its exact traveling wave solutions," Abstract and Applied Analysis, vol. 2013, Article ID 656297, 15 pages, 2013.

[9] J. Li, J. Wu, and H. Zhu, "Traveling waves for an integrable higher order KdV type wave equations," International Journal of Bifurcation and Chaos in Applied Sciences and Engineering, vol. 16, no. 8, pp. 2235-2260, 2006.

[10] J.-b. Li, "Dynamical understanding of loop soliton solution for several nonlinear wave equations," Science in China A, vol. 50, no. 6, pp. 773-785, 2007.

[11] S. A. Khuri, "Soliton and periodic solutions for higher order wave equations of KdV type. I," Chaos, Solitons \& Fractals, vol. 26, no. 1, pp. 25-32, 2005.

[12] Y. Long, W. Rui, and B. He, "Travelling wave solutions for a higher order wave equations of KdV type. I," Chaos, Solitons \& Fractals, vol. 23, no. 2, pp. 469-475, 2005.

[13] V. Marinakis, "Integrable third order equations of the KdV type," Journal of Mathematical Sciences, vol. 5, no. 2, pp. 317-332, 2010.

[14] V. Marinakis, "Higher-order equations of the KdV type are integrable," Advances in Mathematical Physics, vol. 2010, Article ID 329586, 5 pages, 2010.

[15] M. Gandarias and M. S. Bruzon, "Conservation laws for a third order equation by using free software MAXIMA," in Recent Researches in Engineering Education and Software Engineering, pp. 48-51, WSEAS Press, 2012.

[16] X. Li and M. Wang, "A sub-ODE method for finding exact solutions of a generalized $\mathrm{KdV}-\mathrm{mKdV}$ equation with high-order nonlinear terms," Physics Letters A, vol. 361, no. 1-2, pp. 115-118, 2007.

[17] A.-M. Wazwaz, "New sets of solitary wave solutions to the KdV, $\mathrm{mKdV}$, and the generalized KdV equations," Communications in 
Nonlinear Science and Numerical Simulation, vol. 13, no. 2, pp. 331-339, 2008.

[18] R. R. Kumar, K. T. Chong, and J. H. Park, "Exact null controllability of KdV-Burgers equation with memory effect systems," Abstract and Applied Analysis, vol. 2012, Article ID 531659, 20 pages, 2012.

[19] W. Zhang and X. Li, "Approximate damped oscillatory solutions for generalized KdV-Burgers equation and their error estimates," Abstract and Applied Analysis, vol. 2011, Article ID 807860, 26 pages, 2011.

[20] J.-M. Kim and C. Chun, "New exact solutions to the KdVBurgers-Kuramoto equation with the Exp-function method," Abstract and Applied Analysis, vol. 2012, Article ID 892420, 10 pages, 2012.

[21] W. Rui, B. He, Y. Long, and C. Chen, "The integral bifurcation method and its application for solving a family of thirdorder dispersive PDEs," Nonlinear Analysis: Theory, Methods \& Applications, vol. 69, no. 4, pp. 1256-1267, 2008. 


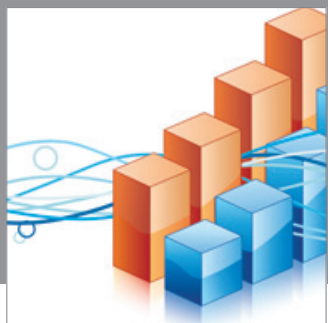

Advances in

Operations Research

mansans

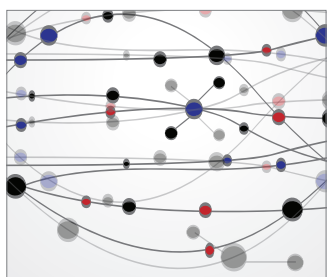

The Scientific World Journal
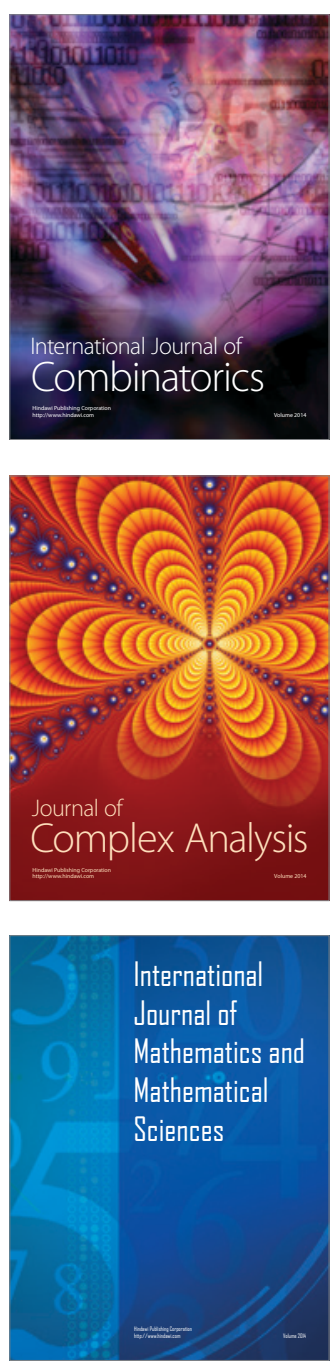
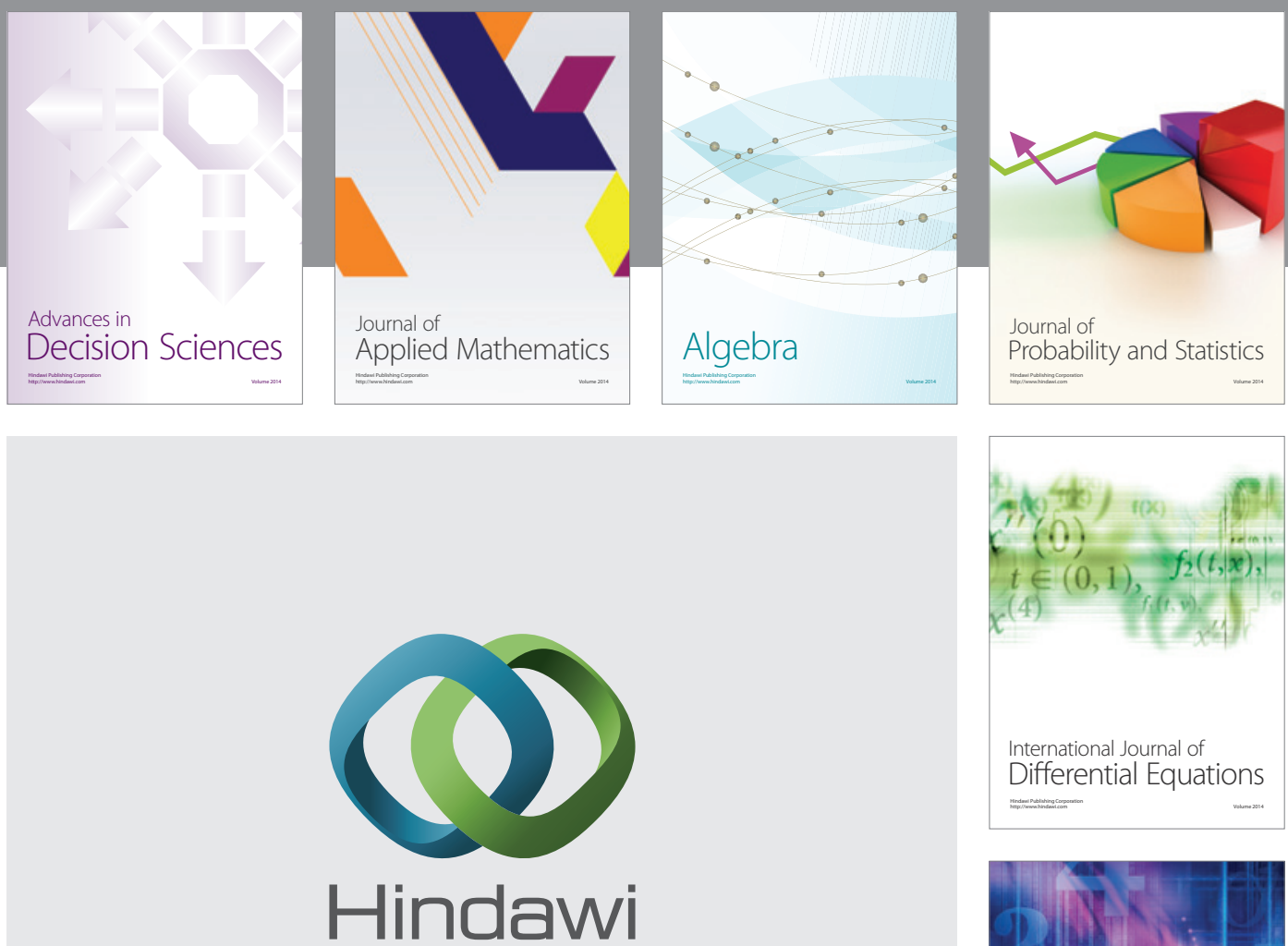

Submit your manuscripts at http://www.hindawi.com
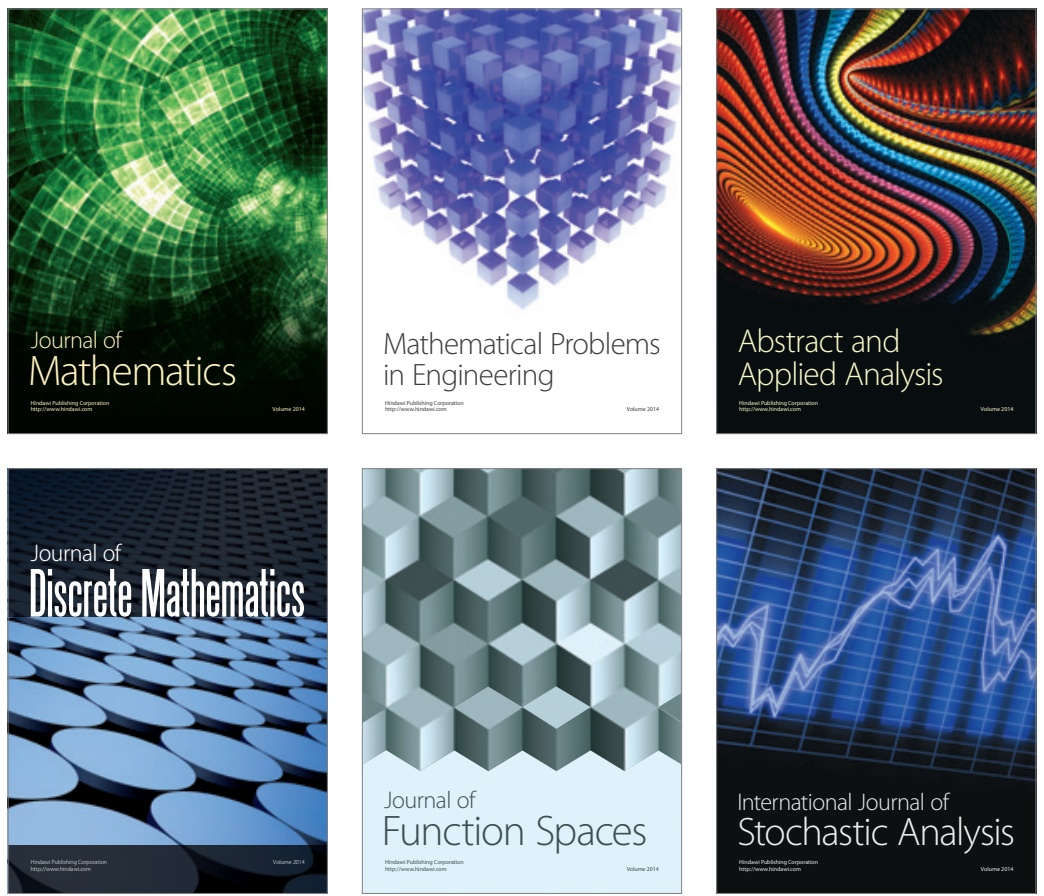

Journal of

Function Spaces

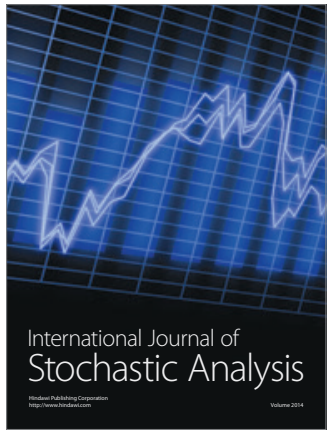

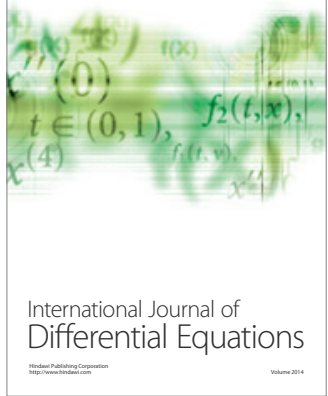
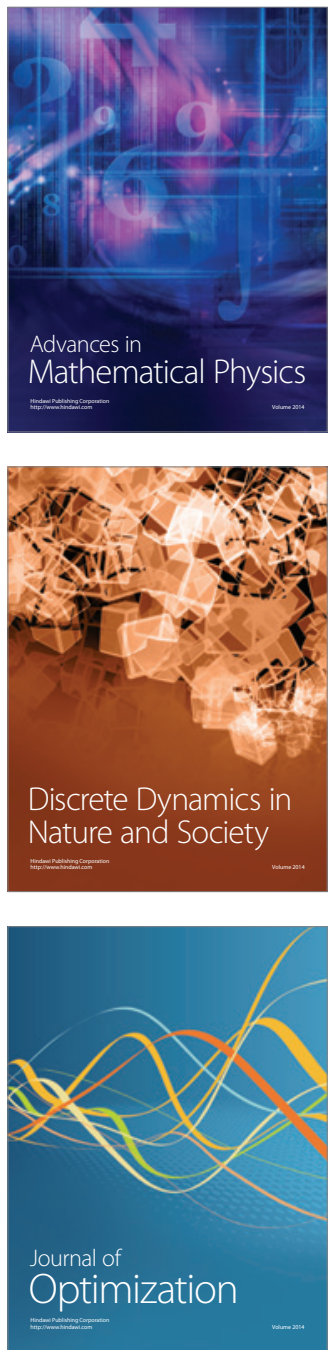